\title{
Experimental Finding
}

National Cancer Institute

\section{Source}

National Cancer Institute. Experimental Finding. NCI Thesaurus. Code C76124.

An interpretation of results of an experiment. 